\title{
Functionalized graphene oxide for clinical glucose biosensing in urine and serum samples
}

\author{
This article was published in the following Dove Press journal: \\ International Journal of Nanomedicine \\ 17 December 2012 \\ Number of times this article has been viewed
}

\author{
Murugan Veerapandian' \\ Yeong-Tai Seo ${ }^{2}$ \\ Hyunkyung Shin ${ }^{3}$ \\ Kyusik Yun' \\ Min-Ho Lee ${ }^{4}$ \\ 'Department of Bionanotechnology, \\ Gachon University, Gyeonggi-Do, \\ ${ }^{2}$ School of Electrical Engineering and \\ Computer Science, Seoul National \\ University, Seoul, ${ }^{3}$ Department \\ of Mathematics and Information, \\ Gachon University, Gyeonggi-Do, \\ ${ }^{4}$ Korea Electronics Technology \\ Institute, Medical IT Technology, \\ Gyeonggi-Do, Republic of Korea
}

Correspondence: Kyusik Yun Department of Bionanotechnology, Gachon University, Gyeonggi-do, Republic of Korea

Tel +823 I 7508753

Fax +82 3I 750 88I 9

Email ykyusik@gachon.ac.kr

Min-Ho Lee

Medical IT Technology,

Korea Electronics Technology Institute,

Gyeonggi-do, Republic of Korea

Tel +82 3। 7897549

Email mhlee@keti.re.kr

\begin{abstract}
A novel clinical glucose biosensor fabricated using functionalized metalloid-polymer (silver-silica coated with polyethylene glycol) hybrid nanoparticles on the surface of a graphene oxide nanosheet is reported. The cyclic voltammetric response of glucose oxidase modification on the surface of a functionalized graphene oxide electrode showed a surface-confined reaction and an effective redox potential near zero volts, with a wide linearity of $0.1-20 \mathrm{mM}$ and a sensitivity of $7.66 \mu \mathrm{A} \mathrm{mM}^{-1} \mathrm{~cm}^{-2}$. The functionalized graphene oxide electrode showed a better electrocatalytic response toward oxidation of $\mathrm{H}_{2} \mathrm{O}_{2}$ and reduction of oxygen. The practical applicability of the functionalized graphene oxide electrode was demonstrated by measuring the peak current against multiple urine and serum samples from diabetic patients. This new hybrid nanoarchitecture combining a three-dimensional metalloid-polymer hybrid and two-dimensional graphene oxide provided a thin solid laminate on the electrode surface. The easy fabrication process and retention of bioactive immobilized enzymes on the functionalized graphene oxide electrode could potentially be extended to detection of other biomolecules, and have broad applications in electrochemical biosensing.
\end{abstract}

Keywords: clinical diagnostics, glucose biosensor, metalloid-polymer nanoparticles, glucose oxidase, cyclic voltammetry

\section{Introduction}

Development of novel biosensors for early detection of specific analytes and selfmonitoring of disease states is widely recognized as an effective method for diagnosis of metabolic disorders such as diabetes. ${ }^{1,2}$ According to a recent International Diabetes Federation report, 380 million people are expected to suffer from diabetes by $2025 .^{3}$ Although a large number of glucose sensors, such as urine glucose meters and blood glucose sensors, have been developed and are commercially available, there is growing interest in developing an advanced biosensor system serving as a reusable self-monitoring device with improved analytical performance, ability to resist interference, and possible multiple functionalities in the same device. Of the biosensor methods available, electrochemical analysis has been shown to have inherent benefits of simplicity, high sensitivity, good selectivity, easy integration, and relatively low cost. ${ }^{4-6}$ Therefore, different chemically modified electrodes have been developed for biomolecular analysis, environmental monitoring, and analysis of food quality. ${ }^{7-11}$ Functional modification of electrode substrates for the construction of electrochemical biosensors having improved analytical performance with respect to other biosensor designs is highly desirable for developing potential device applications. ${ }^{10,11}$ In particular, electrodes that are modified or functionalized by nanoscale materials 
provide a suitable microenvironment for immobilization of biomolecules which can retain their biological activity and enhance electron transfer between biomolecules and the electrode. ${ }^{11-13}$ This paper reports the results of one such advanced nanomaterial-functionalized electrode for sensitive electrochemical biosensing of clinical glucose samples.

Graphene oxide nanosheets have emerged as a novel material with an expanded active surface area, good dispersibility in water at the molecular level, good biocompatibility, and a potentially low manufacturing cost..$^{14-17}$ The unique two-dimensional sheet-like nanostructure, high surface-tovolume ratio, and physicochemical properties of graphene oxide make it a promising candidate for constructing electrochemical sensors and biosensors. According to the widely accepted Lerf-Klinowski model, graphene oxide platelets have chemically reactive oxygen functionality, including carboxylic acid groups at their edges and epoxy and hydroxyl groups on the basal planes. Therefore, chemical functionalization of graphene oxide can be mediated by orthogonal reactions of these groups to functionalize one site selectively over another. ${ }^{18}$ Thus, when such chemically reactive components are modified with other active nanoparticles; a novel three-dimensional hybrid nanoarchitecture with features of increased binding affinity towards bioactive molecules and electrode substrates is achieved. ${ }^{19}$

Several reports of carbon composite materials, such as graphene-gold nanoparticles-chitosan hybrid film and glucose oxidase-assembled graphene and graphene oxidePrussian blue hybrid film for glucose biosensing have been published. ${ }^{19-21}$ Although graphene-based electrodes and other electrodes modified by composite materials have demonstrated their utility as electrochemical glucose biosensors in phosphate-buffered saline, and serum and urine samples, ${ }^{3,19-22}$ to the best of our knowledge, their use for detection of glucose in urine and serum samples from diabetic patients using a functionalized electrode has not yet been studied. Furthermore, the graphene-based composites utilized so far for electrochemical studies have been fabricated by physical modification ${ }^{19}$ and are not chemically bonded or functionalized, suggesting lack of long-term binding affinity and stability problems between nanoparticles and graphene oxide during electrochemical analysis. Therefore, it would be worthwhile developing a chemically stable functionalized graphene oxide material with the ability to determine urinary and plasma glucose levels. In addition, studying the direct electrochemical response of such a hybrid material with the potential to act as a biosensing platform would be of interest in a number of interdisciplinary fields.
Here we describe a novel functionalized graphene oxide (FGO)-based electrochemical biosensor for clinical detection of glucose in different types of physiological samples. The impetus for development of this device arose from the established use of metalloid-polymer nanostructures, including thin solid laminates and active materials used in sensor studies. ${ }^{23-25}$ We used metalloid-polymer hybrid nanoparticles consisting of silver-silica $\left(\mathrm{Ag} @ \mathrm{SiO}_{2}\right)$ and polyethylene glycol (PEG) with an average particle diameter of $12 \pm 3 \mathrm{~nm}$ to functionalize the surface of graphene oxide. The nanoparticles were first silanized by reacting them with 3 -aminopropyltriethoxysilane and then covalently reacting them with the oxygen group of graphene oxide. Spectroscopic investigations revealed that the carboxylic acid groups of graphene oxide were directly involved in the covalent reaction with silanized metalloid-polymer hybrid nanoparticles. The electrochemical efficiency of the novel FGO-modified electrode was superior to that of a commercial gold-printed circuit board (Au-PCB) electrode. The synergistic features of the nanohybrid (containing metal, nonmetal, and polymer particles) on the surface of graphene oxide are believed to be the factors involved in its direct electron transfer properties, by providing the smallest difference of oxidation and reduction potential near zero volts. The fabricated FGO material was developed to test the possibility of electrochemical biosensing of glucose molecules in phosphate-buffered saline and in urine and serum samples from diabetic patients.

\section{Materials and methods Materials}

The precursor materials required for the synthesis of the metalloid-polymer hybrid nanoparticles (MPHs), namely silver nitrate $\left(\mathrm{AgNO}_{3}\right)$, tetraethoxysilane, sodium borohydride, and ammonium hydroxide, as well as the 3-aminopropyltriethoxysilane needed for functionalization of graphene oxide were purchased from Sigma-Aldrich (St Louis, MO). PEG (number average molecular weight $10,000 \mathrm{~g} / \mathrm{mol}$ ), expandable graphite powder, $\mathrm{D}(+)$ glucose, and glucose oxidase from Aspergillus niger were also obtained from SigmaAldrich. Sulfuric acid, potassium permanganate, hydrogen peroxide, and hydrochloric acid were obtained from Daejung Chemicals and Metals Ltd, Gyeonggi-do, South Korea. Milli-Q water with resistance greater than $18 \mathrm{M} \Omega$ was used in all experiments. All chemicals were of analytical grade and used as received without further purification.

The urine and serum samples from diabetic patients utilized in the biosensor study were kindly provided by Bundang Seoul National University Hospital, Seoul, South Korea. 
All experiments were performed according to a protocol approved by the institutional review committee at Seoul National University Bundang Hospital. Before involvement in any study-related procedures, all patients gave their written informed consent. Table S1 provides the patient details and their respective serum glucose concentrations as measured by a commercially available glucose sensor.

\section{Instrumentation}

High-resolution transmission electron microscopic imaging of graphene oxide and the FGO-modified MPHs was performed using a Cs-corrector-equipped Titan 80-300 device (FEI, Hillsboro, OR) operated at $300 \mathrm{kV}$. The samples used for the measurements were prepared by drop-casting $5-10 \mu \mathrm{L}$ of graphene oxide $(0.1 \mathrm{mg} / \mathrm{mL})$ or FGO suspension onto the surface of a copper grid, and allowing the solvent to evaporate before measurement. Ultraviolet-visible absorbance spectra for aqueous solutions of graphene oxide and FGO were measured using a Varian Cary 50 spectrophotometer. The average particle size distribution of the as-prepared MPHs was studied (dispersing the dried particles in anhydrous ethanol) using the electrophoretic light scattering method with an ELS-8000 (Otsuka Electronics Co, Ltd, Osaka, Japan). Prior to measurement, colloidal solutions were filtered through a Millipore membrane (pore size $0.2 \mu \mathrm{m}$ ) three times and then introduced into the cell. The chemical structure and functional groups of graphene oxide were observed before and after functionalization with MPHs using a Fourier transform-infrared spectrophotometer (Nicolet 6700) and a $\mathrm{KBr}$ disk at a resolution of $4 \mathrm{~cm}^{-1}$. Raman spectra for pure graphene oxide and FGO were recorded using a Micro Raman system (Ramboss-500i) with a $50 \times$ objective lens at room temperature, a $633 \mathrm{~nm} \mathrm{He}-\mathrm{Ne}$ laser beam, and 1800 lines per mm grating. Aqueous dispersions of graphene oxide and of FGO were drop-casted onto a clean glass substrate $(2.0 \times 2.0 \mathrm{~cm})$ and the solvent allowed to evaporate at ambient temperature before carrying out the measurements. Cyclic voltammogram (CV) measurement for the commercial Au-PCB electrode modified with graphene oxide and/or FGO-glucose oxidase (GOx) was done using a VersaSTAT 3 in a three-electrode configuration, with the customized Au-PCB used as the working electrode, and a platinum wire and an $\mathrm{Ag} / \mathrm{AgCl}$ electrode used as the counter and reference electrodes, respectively.

\section{Synthesis of $\mathrm{Ag} @ \mathrm{SiO}_{2}-\mathrm{PEG}$ (MPHs)}

Metalloid-polymer hybrid nanoparticles composed of $\mathrm{Ag} @$ $\mathrm{SiO}_{2}$-PEG (average size $12 \pm 3 \mathrm{~nm}$ ) were synthesized using an ultrasonochemical approach, following our previous report. ${ }^{24}$
Briefly, $150 \mu \mathrm{L}$ of a precursor $30 \mathrm{mM} \mathrm{AgNO}_{3}$ aqueous solution was added to a reaction vessel containing $12 \mathrm{~mL}$ of the stabilizing agent (PEG, number average molecular weight $10,000 \mathrm{~g} / \mathrm{mol}$ ) and $300 \mu \mathrm{L}$ of $30 \mathrm{mM}$ sodium borohydride as a reducing agent. Under controlled conditions, including probe temperature $\left(65^{\circ} \mathrm{C}\right)$, amplitude $(35 \%)$, and pulser on-off cycle (5-10 seconds), the reaction mixture was ultrasonicated for 15 minutes to form the initial silver core. Following this, the desired amounts of aqueous tetraethoxysilane $(150 \mu \mathrm{L}$, $30 \mathrm{mM})$ and ammonium hydroxide $(300 \mu \mathrm{L})$ were added. The reaction vessel containing the precursor solutions of metal, nonmetal, PEG, and reducing agent were again subjected to ultrasonication for 30 minutes to ensure a complete reduction reaction and formation of the hybrid structure. The hybrid particles were isolated from the resulting colloidal solution by centrifugation, washed twice with deionized water and ethanol, and then used for the functionalization experimentation.

\section{Functionalization of MPHs onto graphene oxide}

The brownish colloidal suspensions of graphene oxide nanosheets utilized in the current experiment were prepared by vigorous oxidation of graphite powder, according to a modified Hummers method. ${ }^{15,26}$ Functionalization of MPHs onto the surface of graphene oxide was then achieved by a two-step process, following a recent report. ${ }^{25}$ Briefly, $200 \mu \mathrm{L}$ of MPHs at a concentration of $5 \mathrm{mg} / \mathrm{mL}$ and $40 \mu \mathrm{L}$ of 3-aminopropyltriethoxysilane ( $30 \mu \mathrm{L} / \mathrm{mL}$ in $\mathrm{C}_{2} \mathrm{H}_{5} \mathrm{OH}$ ) was added to a vial containing $3 \mathrm{~mL}$ of anhydrous ethanol and magnetically stirred at $800 \mathrm{rpm}$ and room temperature for 10 hours. Next, an aqueous solution of $200 \mu \mathrm{L}$ graphene oxide (concentration $2.5 \mathrm{mg} / \mathrm{mL}$ ) was added and magnetically stirred at $800 \mathrm{rpm}$ for another 10 hours to ensure a covalent reaction between the silanized MPHs and graphene oxide. After completion of the reaction process, the MPHs functionalized with graphene oxide sheets were separated by centrifugation at 12,000 rpm for 30 minutes, washed three times with ethanol, and used for construction of the biosensor platform.

\section{Fabrication of Au-PCB-FGO electrode for biosensor platform}

After performing sequential washing with anhydrous ethanol, acetone, and deionized water, the Au-PCB working electrode (approximately $1 \mathrm{~mm}$ in diameter) underwent a surface immobilization procedure starting with oxygen plasma treatment for 2 minutes. Several experimental optimizations were carried out to obtain a suitable working potential 
range (from -0.15 to $+0.25 \mathrm{~V}$ ) and an optimal $\mathrm{CV}$ response. Typically, $4 \mu \mathrm{L}$ of aqueous graphene oxide and/or FGO suspension $(2 \mathrm{mg} / \mathrm{mL}$ ) was drop-casted onto the Au-PCB electrode and allowed to evaporate at ambient temperature for one hour. The resulting substrate was then coated with $4 \mu \mathrm{L}$ of glucose oxidase $(5 \mathrm{mg} / \mathrm{mL}$ in phosphate-buffered solution), refrigerated at $4^{\circ} \mathrm{C}$ for one hour, and the resulting glucose oxidase-modified Au-PCB electrode modified with graphene oxide (Au-PCB-GO-GOx) and/or with functionalized graphene oxide (Au-PCB-FGO-GOx) was used for electrochemical characterization. A reproducible $\mathrm{CV}$ was obtained under steady-state conditions after about five cycles. Unless otherwise stated, all CV measurements were recorded at a constant scan rate of $50 \mathrm{mV}$ per second and a potential range from -0.15 to $+0.25 \mathrm{~V}$.

Three types of electrochemical $\mathrm{CV}$ were recorded for the Au-PCB-GO-GOx and Au-PCB-FGO-GOx electrodes, including for different concentrations and volumes of $\mathrm{D}(+)$ glucose and clinical glucosuric samples in phosphatebuffered solution (50 mL, pH 7.4), respectively, and also serum samples from diabetic patients modified with Au-PCBFGO-GOx in phosphate-buffered solution (50 mL, pH 7.4).
Phosphate-buffered solution was used as the common electrolyte for all electrochemical measurements. The solution was prepared by dissolving a phosphate-buffered saline tablet (Sigma-Aldrich) in $200 \mathrm{~mL}$ of distilled water, giving a final concentration of $0.0027 \mathrm{M}$ potassium chloride and $0.137 \mathrm{M}$ sodium chloride, with a $\mathrm{pH} 7.4$ at $25^{\circ} \mathrm{C}$.

\section{Results and discussion Optical absorbance, morphology, and Raman spectral analysis of graphene oxide and FGO}

The general strategy for functionalization of MPHs onto the surface of graphene oxide can be explained by two important surface chemical reactions, ie, amidation by a carboxylic group from graphene oxide and silanized MPHs and nucleophilic attack at the $\alpha$-carbon by the amine groups on silanized MPHs. ${ }^{25}$ Figure 1A shows the ultraviolet-visible absorbance spectrum of graphene oxide synthesized by the modified Hummers method. ${ }^{15,26}$ The wavelength of maximum absorbance for graphene oxide was found at $235 \mathrm{~nm}$, which corresponds to the characteristic $\pi-\pi^{*}$ electron transitions of
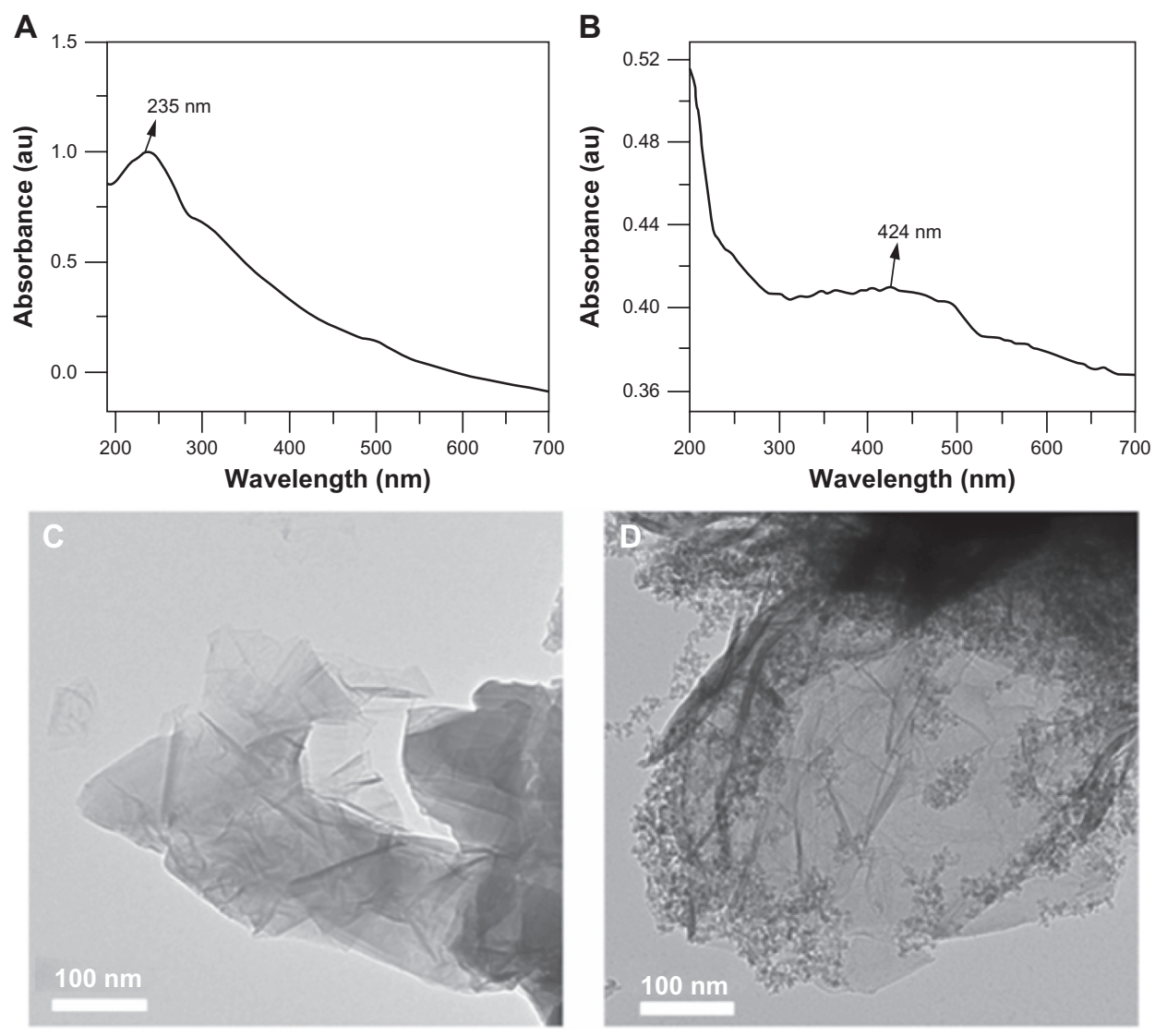

Figure I Ultraviolet-visible absorbance spectrum (A) graphene oxide and (B) functionalized graphene oxide, and transmission electron microscopic images of (C) graphene oxide and (D) functionalized graphene oxide. 
the aromatic $\mathrm{C}-\mathrm{C}$ bonds. ${ }^{16,26}$ As shown in Figure S1A, MPHs in their normal state have a $\lambda_{\text {max }}$ at $418 \mathrm{~nm}$, in agreement with previous reports for MPHs. ${ }^{23-25}$ Surface functionalization of graphene oxide with MPHs resulted in a shifted peak at $424 \mathrm{~nm}$ with a broadened shape (see Figure 1B). In general, transition metal colloidal nanoparticles, such as those containing silver, have a well known surface plasmon resonance depending on particle size and shape. Surface plasmon resonance is seen due to the collective oscillations of conducting electrons from the sample species. ${ }^{27}$ The optical absorption spectrum of metal nanoparticles is always dominated by surface plasmon resonance, leading to a distinct shift to either red or blue, depending on the dielectric properties of the surrounding matrix or environmental conditions. ${ }^{24,27}$ In this study, the final hybrid FGO would be expected to have the two characteristics of optical absorbance, such as $\pi-\pi^{*}$ electron transitions from the aromatic $\mathrm{C}-\mathrm{C}$ of graphene oxide, and surface plasmon resonance related to the surfacemodified silver nanostructures. However, the peak obtained for FGO showed a broadened signal centered at $424 \mathrm{~nm}$. Possible reasons for this observation could be the surface chemical modification of the silver core by nonmetal silica and the PEG polymer layer. In addition, FGO has MPHs on its surface promoting strong interaction between graphene oxide and MPHs, that may influence the width and overlap between graphene oxide optical absorbance and the MPH peak (Figure 1B). ${ }^{25}$

High-resolution transmission electron micrographs were obtained to verify the structural characteristics of graphene oxide before and after functionalization with MPHs. Normal graphene oxide (Figure 1C) had the appearance of a wrinkled sheet-like structure, whereas FGO (Figure 1D) had a surface-functionalized nanoparticle structure. Graphene oxide is a two-dimensional nanosheet material with a large active surface area and good biocompatibility, and is used widely as a building block for surface and interface studies. Functionalization of three-dimensional heterogeneous MPHs (containing a silver metal core, a silica shell, and PEG as polymer layer, ie, a three-in-one system) on the surface of graphene oxide provides an advanced three-dimensional hierarchical structure with multifunctional features. ${ }^{25}$

As shown in Figure 2, Raman spectral analysis was used to observe the ordered and disordered crystal structures of graphene oxide and FGO. The Raman spectrum of graphene oxide showed a characteristic $\mathrm{G}$ band at $1595.7 \mathrm{~cm}^{-1}$ assigned to the $\mathrm{E}_{2 \mathrm{~g}}$ phonon mode of $\mathrm{sp}^{2}$-hybridized carbon atoms, and a D-band at $1345.7 \mathrm{~cm}^{-1}$ assigned to the vibrations of carbon atoms with dangling bonds in plane terminations

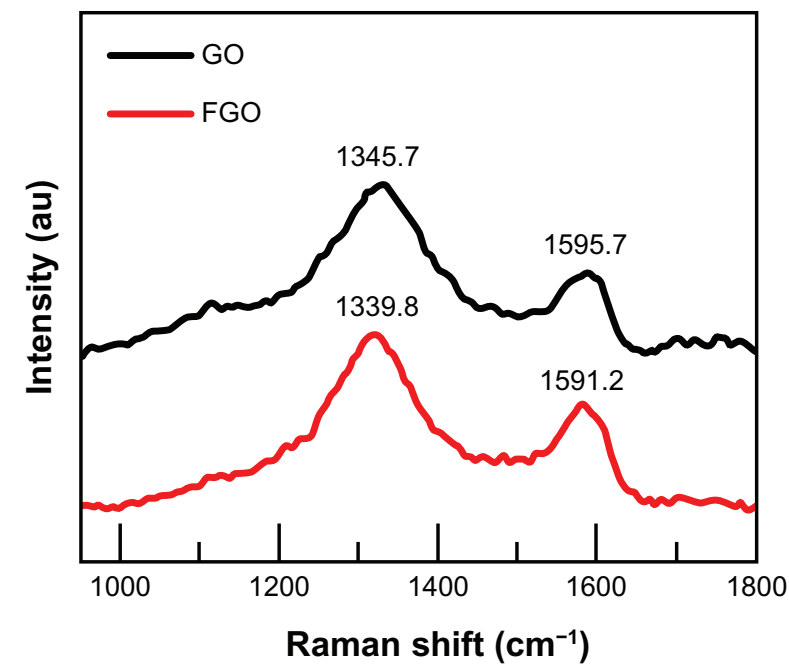

Figure 2 Raman spectrum of graphene oxide and functionalized graphene oxide. Abbreviations: GO, graphene oxide; FGO, functionalized graphene oxide.

of disordered graphite, particularly at the edges. ${ }^{15,16,25}$ After functionalization of graphene oxide by silanized MPHs, the $\mathrm{D}$ bands and $\mathrm{G}$ bands were blue-shifted at $1339.8 \mathrm{~cm}^{-1}$ and $1591.2 \mathrm{~cm}^{-1}$, respectively. Furthermore, the D peak intensity $\left(I_{(\mathrm{D})}\right)$ was increased in the FGO spectrum compared with that for normal graphene oxide, indicating that our functionalization process had modified the lattice structure of the graphene oxide sheets. The ratio of $\mathrm{D}$ band to $\mathrm{G}$ band intensity $\left(I_{(\mathrm{D})} / I_{(\mathrm{G})}\right)$ for FGO was calculated to be slightly larger than that for graphene oxide, confirming that functionalization of graphene oxide with silanized MPHs significantly influenced its structure. These results are in good agreement with previous reports on functionalization of graphene oxide sheets. ${ }^{25,28}$ The structural integrity of individual graphene oxide, MPHs, and the combined functional nanosheets were separately characterized for their stretching and bending vibrations by Fourier transform-infrared spectroscopy and the results are included as supporting information (see Figure S2).

\section{Construction of biosensor platform for glucose detection}

As shown in Figure 3, graphene oxide or FGO was initially immobilized on the surface of the commercial Au-PCB electrode, followed by immobilization of glucose oxidase solution onto the surface of the Au-PCB-GO and/or AuPCB-FGO substrate by drop-casting. Of note, the electrode substrate utilized in the current study differs from the conventional glassy carbon electrode or indium tin oxide electrode. It has 16 distinct patterns of gold substrate (each with a surface area of about $1 \mathrm{~mm}$ in diameter) on a printed circuit board electrode (fabricated by an electroplating method), 


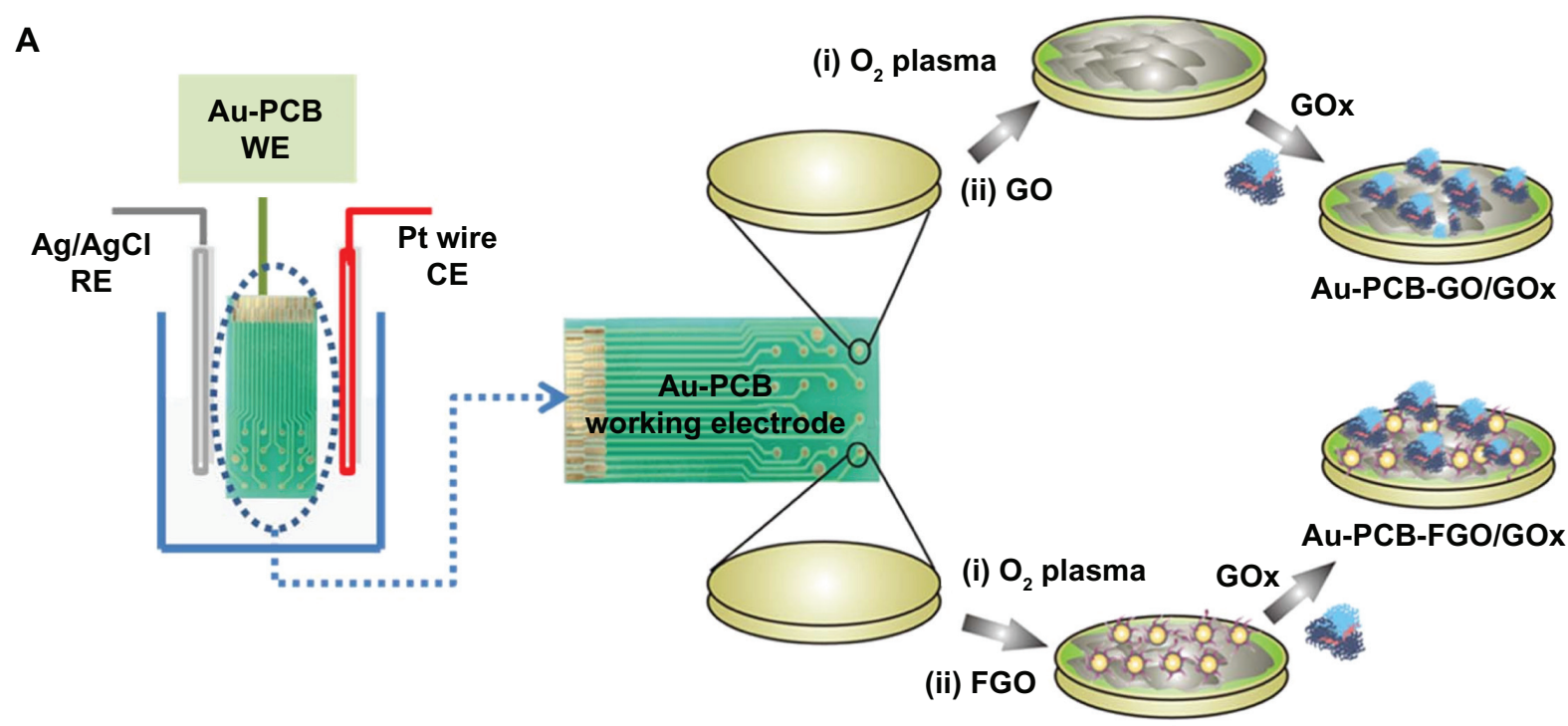

B

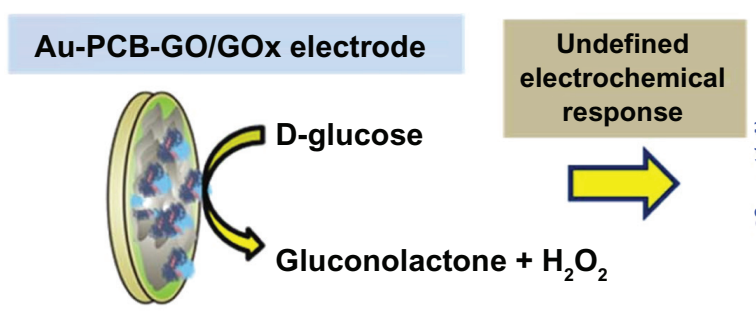

\section{Au-PCB-FGO/GOx electrode}

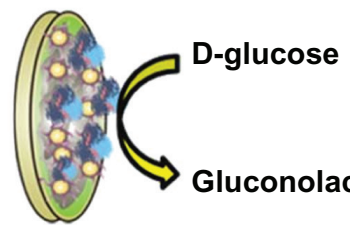

Cyclic voltammogram
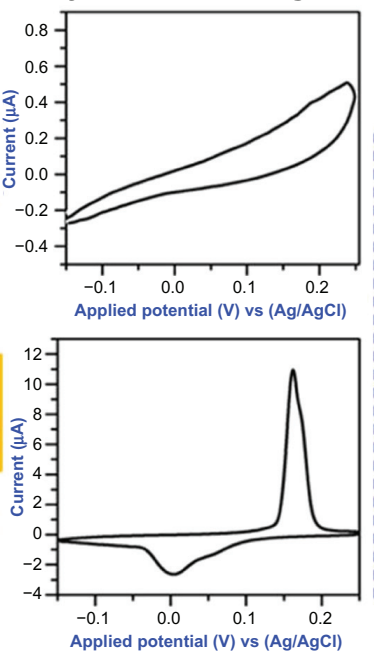

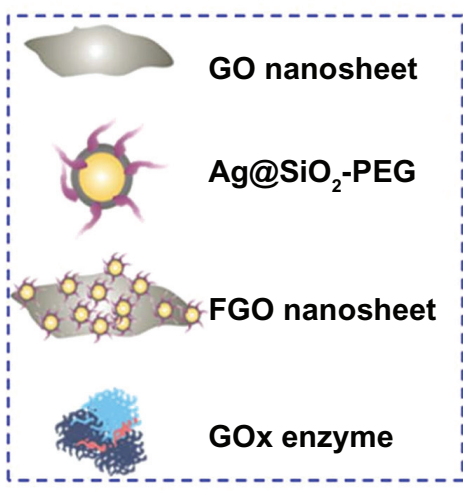

Figure 3 (A) Illustration of the electrochemical setup under electrolyte solution. Dotted circle depicts the digital image of Au-PCB working electrode and the process of glucose oxidase modification at two different stages, ie, Au-PCB-GO and Au-PCB-FGO biosensor platform, respectively. (B) Enzymatic reaction at the electrode interface and its respective cyclic voltammogram.

Abbreviations: $\mathrm{Ag} / \mathrm{AgCl}$, silver/silver chloride; RE, reference electrode; Pt, platinum; $\mathrm{CE}$, counter electrode; Au-PCB, gold printed circuit board electrode; WE, working electrode; Au-PCB-GO, gold printed circuit board electrode modified with graphene oxide; Au-PCB-FGO, gold printed circuit board electrode modified with functionalized graphene oxide; GOx, glucose oxidase; $\mathrm{Ag@SiO}{ }_{2}-\mathrm{PEG}$, silver-silica coated with polyethylene glycol; FGO, functionalized graphene oxide; GO, graphene oxide.

with the potential for multiple surface modifications using active moieties or enzymes for sensor studies. Depending on the location of the surface modification, the gold substrate is fixed with a wire to connect the electrochemical system. The present Au-PCB electrode (customized as a working electrode) can be reused after performing a sequential wash with an alcoholic solvent or acetone in an ultrasonication bath to remove the immobilized materials. PCB electrodes are more advantageous than thin-film electrodes in terms of cost, design features, fabrication, large-scale production, reliability, and electronic integration. ${ }^{29}$ Using PCB technology, it is possible to design low-resistance electrodes on insulator substrates which can withstand larger currents than a thin-film electrode. $^{29}$

After performing the necessary washing step as described in the experimental section, oxygen plasma treatment was performed to modify the gold substrate into a hydrophilic one for enhancing immobilization of graphene oxide. In general, graphene oxide possesses chemically reactive oxygen functional groups at the edges and basal planes of a sheet-like nanostructure, making it suitable for several types of chemical functionalization. We used CVs to analyze the electrochemical response of the fabricated electrodes. Figure 4A shows the fundamental electrochemical responses of glucose oxidase- 


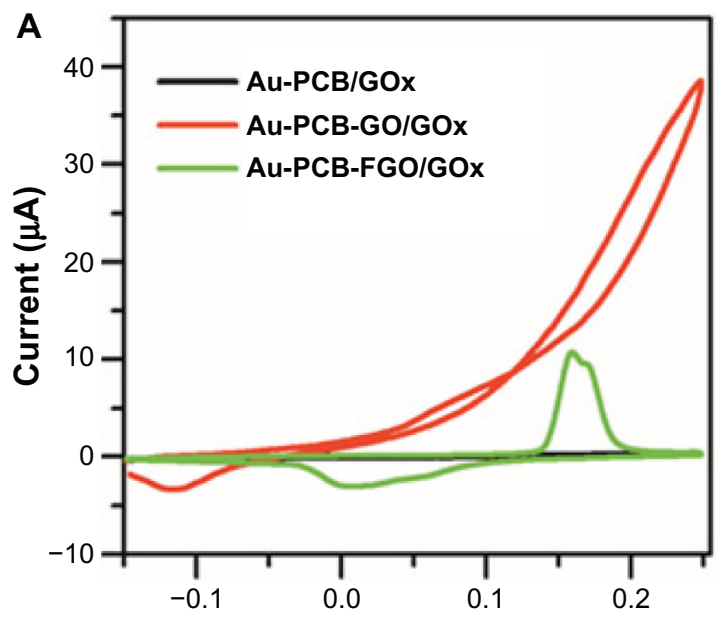

Applied potential (V) vs Ag/AgCl

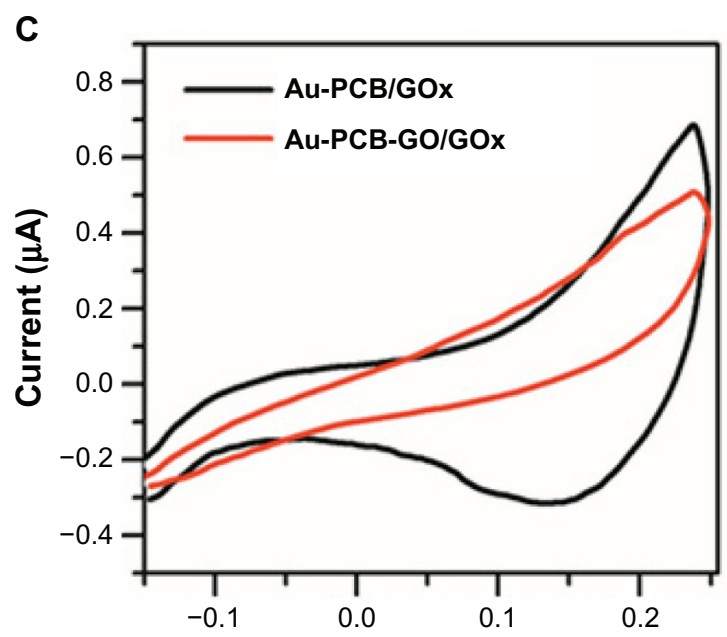

Applied potential (V) vs Ag/AgCl

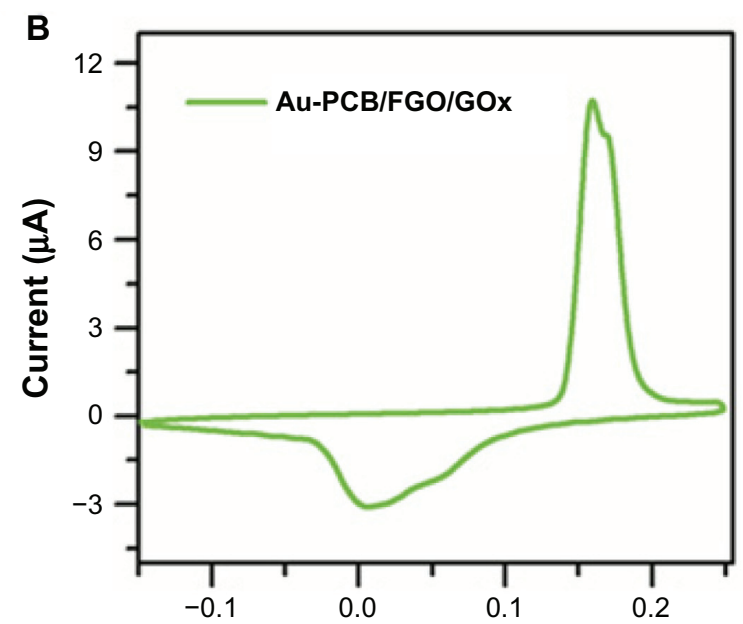

Applied potential (V) vs Ag/AgCl

\section{D}

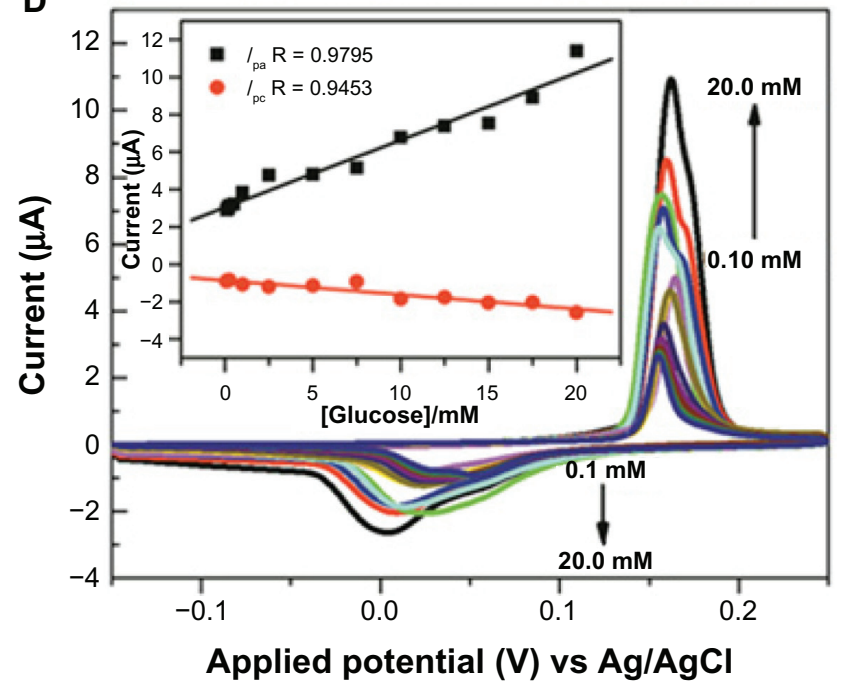

Figure 4 (A) CVs of GOx-modified Au-PCB (black trace), Au-PCB-GO (red trace), and Au-PCB-FGO electrode in PBS buffer (pH 7.4). (B) Focused CV curve of Figure 4A (green trace) GOx-modified Au-PCB-FGO electrode. (C) CVs of bare Au-PCB/GOx (black trace) and Au-PCB-GO/GOx (red trace) versus 20 mM D(+) glucose in PBS $(\mathrm{pH}$ 7.4). (D) $\mathrm{CVs}$ of Au-PCB-FGO-GOx versus $\mathrm{D}(+)$ glucose in PBS buffer at different concentration ranges from $20 \mathrm{mM}$ to $0.1 \mathrm{mM}$, and the inset shows the corresponding plot of peak currents against molar concentrations of glucose.

Notes: Scan rate: $50 \mathrm{mV} / \mathrm{s}$. All the individual CV responses were measured by different electrodes prepared appropriately.

Abbreviations: CVs, cyclic voltammograms; GOx, glucose oxidase; Au-PCB, gold printed circuit board electrode; Au-PCB-GO, gold printed circuit board electrode modified with graphene oxide; Au-PCB-FGO, gold printed circuit board electrode modified with functionalized graphene oxide; Au-PCB-FGO-GOx, gold printed circuit board electrode modified with functionalized graphene oxide and glucose oxidase; PBS, phosphate-buffered saline; $\mathrm{Ag} / \mathrm{AgCl}$, silver/silver chloride.

modified Au-PCB, Au-PCB-GO, and Au-PCB-FGO in phosphate-buffered solution. As observed in Figure 4B, the CV curve for Au-PCB-FGO-GOx (Figure 4A, green trace) exhibits the well-defined redox peak (corresponding to characteristic cathodic peak potential $\left(E_{\mathrm{pc}}\right)$ and anodic peak potential $\left(E_{\mathrm{pa}}\right)$ centered at $0.158 \mathrm{~V}$ and at $0.006 \mathrm{~V}$, respectively. In contrast, glucose oxidase-modified bare Au-PCB and $\mathrm{Au}-\mathrm{PCB}-\mathrm{GO}$ do not exhibit any characteristic redox signal either without (Figure 4A) or with D(+)glucose (Figure 4C). The mechanism of redox wave generation from the Au-PCBFGO electrode may involve oxidation and reduction reactions of active MPH on the surface of graphene oxide. ${ }^{25}$ In general, achieving direct electrochemistry of enzymes on conventional electrodes is very difficult due to problems concerning accessibility of active centers, which are located deep within the hydrophobic cavity of the molecule, affecting the local concentration and rate of electron transport. ${ }^{30-32}$ Therefore, researchers have modified the electrode surface with different composites of carbon nanomaterials, such as nanotubes and graphene-based hybrid and metal nanoparticles, to enable significant enhancement of the electron transfer reaction at electrochemical interfaces, such as between enzymes 
and electrodes. ${ }^{31,32}$ It has been demonstrated that the electrical properties of graphene-based materials are stronger than those of other nanomaterials. ${ }^{33}$ Chemical species adsorbed onto the surface of a graphene system act as electron donors or acceptors. ${ }^{34}$ Due to an extended $\pi$-system, graphene materials have charge transfer interactions with electron donor and electron acceptor molecules. ${ }^{35}$ Chemical modification of graphene systems depends strongly on the types of dopants and concentrations used, and their locations within the graphene systems. Chemically modified graphene materials are reported to have enhanced electronic and quantum transport properties. ${ }^{36}$ Similarly, in the current study, chemical functionalization of MPHs on graphene oxide nanosheets allowed a synergistically enhanced electrochemical reaction at the interface between the glucose oxidase enzyme and the electrode than that seen when using pure Au-PCB and Au-PCB-GO. Structurally, the MPH is composed of silver metal as the core and $\mathrm{SiO}_{2}$ as the shell, and is coated by PEG as an inert stabilizing layer to avoid agglomeration of the individual composite particles. ${ }^{24,25}$ The PEG layer on the silversilica composite surface not only prevents particle aggregation but also promotes active immobilization of glucose oxidase on the functionalized electrode surface. For instance, preliminary research has demonstrated the active role of PEG as a spacer molecule, not only enhancing active immobilization of glucose oxidase in an organic matrix at the electrode surface, but also preserving the bioactivity of glucose oxidase, depending on chain length. ${ }^{37}$ Redox wave generation from conventional silver metal nanoparticles or silver-doped composites with silane-modified electrodes has been reported previously in the literature. ${ }^{38,39}$ In short, the mechanism of redox reaction associated with silver-doped aminosilica in phosphate-buffered solution is demonstrated to involve oxidation and reduction potentials at $0.05 \mathrm{~V}$ and $-0.09 \mathrm{~V}$, respectively $\left(\mathrm{Ag}+\mathrm{Cl}^{-} \rightarrow\right.$ $\mathrm{AgCl}$ and $\mathrm{AgCl} \rightarrow \mathrm{Ag}) .{ }^{38}$ On the other hand, when the silversilica composite electrode is immersed in phosphate solution (without $\mathrm{NaCl}$ ), redox reactions are associated with $\mathrm{Ag} \rightarrow$ $\mathrm{Ag}_{2} \mathrm{O}$ and $\mathrm{Ag}_{2} \mathrm{O} \rightarrow \mathrm{Ag}$ at $0.37 \mathrm{~V}$ and $-0.03 \mathrm{~V}^{38}$ Furthermore, studies have revealed that incorporation of silver nanoparticles with aminosilica film improves the electron transfer rate because of the smallest difference in the oxidation and reduction potential. ${ }^{39-41}$

The electrochemical response of the Au-PCB-FGO-GOx electrode against different concentrations of glucose in phosphate-buffered solution ( $\mathrm{pH} 7.4$ ) is shown in Figure 4D. The $\mathrm{CV}$ of the Au-PCB-FGO-GOx electrode is observed to have a significant shift in peak redox potentials and broadness of the peak, especially at an anodic potential (Figure 4B). The reason for the shift in peak currents at the expected redox potential [such as $0.158 \mathrm{~V}\left(E_{\mathrm{pc}}\right)$ and $0.006 \mathrm{~V}\left(E_{\mathrm{pa}}\right)$ ] and the broadness of the anodic peak could reflect concentration-dependent electron transport of molecules and the associated redox reaction at the functionalized electrode surface and interface. Because there were no chemical redox species directly involved in the current study, all the redox reactions observed on $\mathrm{CV}$ come from the MPHs on the surface of the Au-PCB-GO electrode. ${ }^{25}$ Thus, depending on the increase in glucose concentration in the bulk electrolyte, the enzymatic reaction increases the electrochemical redox reaction at the working electrode in a linear manner, such as for oxidation of glucose and reduction of oxygen from the solution. This combined reaction at the working electrode reflects the increased redox wave signal of MPHs with specific broadness in the anodic peak. Earlier reports, such as for metalloid (silver-doped) aminosilica nanocomposites on a fluorine-doped tin oxide electrode $^{39}$ and for a glassy carbon electrode modified by silver nanoparticles, have observed a similar redox wave with specific broadness of the anodic peak in phosphate-buffered solution. ${ }^{41}$ Although showing a shift in peak currents at different potentials, it is clear that the Au-PCB-FGO-GOx electrode in our study ensured the linear relationship of $I_{\mathrm{p}}$ versus molar concentrations of glucose, with a wide linear range from $0.1 \mathrm{mM}$ to $20 \mathrm{mM}$ (R 0.9795 and R 0.9453 for $I_{\mathrm{pc}}$ and $I_{\mathrm{pa}}$, respectively) and a sensitivity of $7.66 \mu \mathrm{A} \mathrm{mM}^{-1} \mathrm{~cm}^{-2}$, suggesting that the synergistic effect from MPHs functionalized with graphene oxide provided the necessary conduction pathways and electron transfer kinetics at the active substrate. Moreover, the sensitivity obtained for this biosensor was much better than that for other reported glucose biosensors, such as poly(3,4-ethylenedioxythiophene)-Prussian blue multiwalled carbon nanotubes $\left(2.67 \mu \mathrm{A} \mathrm{mM}^{-1} \mathrm{~cm}^{-2}\right)^{42}$ and glucose oxidase-modified Prussian blue screen-printed electrodes $\left(3.21 \pm 0.16 \mu \mathrm{A} \mathrm{mM}^{-1} \mathrm{~cm}^{-2}\right) .{ }^{43}$ In addition, the linear range (up to $20 \mathrm{mM}$ ) is much wider than for similar biosensors, such as glucose oxidase immobilized onto a nanocomposite graphene-gold nanoparticle-chitosan film $(10 \mathrm{mM}),{ }^{19}$ graphene $(10 \mathrm{mM}),{ }^{20}$ graphene oxide-Prussian blue hybrid film $(1.2 \mathrm{mM}),{ }^{21}$ silver-DNA hybrid nanoparticles $(1.2 \mathrm{mM}),{ }^{44}$ a copper self-assembled monolayer nanocomposite on a gold substrate $(0.0018 \mathrm{mM}),{ }^{45}$ poly (3,4-etheylenedioxythiophene)Prussian blue multiwalled carbon nanotubes $(10.0 \mathrm{mM}),{ }^{42}$ highly ordered polyaniline nanotubes $(5.5 \mathrm{mM}),{ }^{46}$ a chitosangold nanoparticle hybrid film on a Prussian blue-modified electrode $(1.6 \mathrm{mM}),{ }^{47}$ a highly ordered porous anodic alumina membrane-Prussian blue nanoarray $(8.0 \mathrm{mM}),{ }^{48}$ and a Prussian blue-modified screen-printed electrode $(3.0 \mathrm{mM}){ }^{43}$ 
The diagnosis of diabetes is made on identification of fasting plasma glucose concentrations $\geq 126 \mathrm{mg} / \mathrm{dL}(7.0 \mathrm{mM})$ on at least two occasions. ${ }^{49}$ Our fabricated biosensor is therefore suitable for practical application in the detection of human blood sugar concentrations. The linear response with respect to various molar concentrations of glucose and the change in the peak current at the Au-PCB-FGO-GOx electrode surface indicate that enzymes immobilized on the surface of FGO provide a suitable environment for electron transfer between the glucose analyte and the sensor surface, which in turn detects the $\mathrm{H}_{2} \mathrm{O}_{2}$ obtained during the enzymatic reaction (glucose oxidase: glucose $+\mathrm{O}_{2} \rightarrow$ gluconolactone $+\mathrm{H}_{2} \mathrm{O}_{2}$ ). In the present study, with increasing glucose concentrations (from $0.1 \mathrm{mM}$ to $20 \mathrm{mM}$ ), the peak oxidation currents also increased due to oxidation of the $\mathrm{H}_{2} \mathrm{O}_{2}$ generated, while the peak reduction current decreased due to consumption of $\mathrm{O}_{2}$. FGO-modified electrode has exhibited an enhanced electrocatalytic current and earlier onset of redox potential. Thus, similar to other electrochemical devices it can be a potential candidate for application in glucose detection. ${ }^{19-21}$

\section{Clinical glucose detection}

The fabricated Au-PCB-FGO-GOx biosensor platforms were then utilized for detecting glucose from two types of clinical diabetic samples, namely urine and serum. Interferents such as ascorbic acid or acetaminophen, or adherents such as urea compounds, are critical factors causing difficulty in urinary glucose detection. ${ }^{3}$ After performing preliminary volumetric assessments using 10,60 , and $160 \mu \mathrm{L}$ urine samples from five diabetic patients (see Figure S3), the CV for a sample from a glucosuric patient was selected as the model clinical sample to confirm glucose detection by our

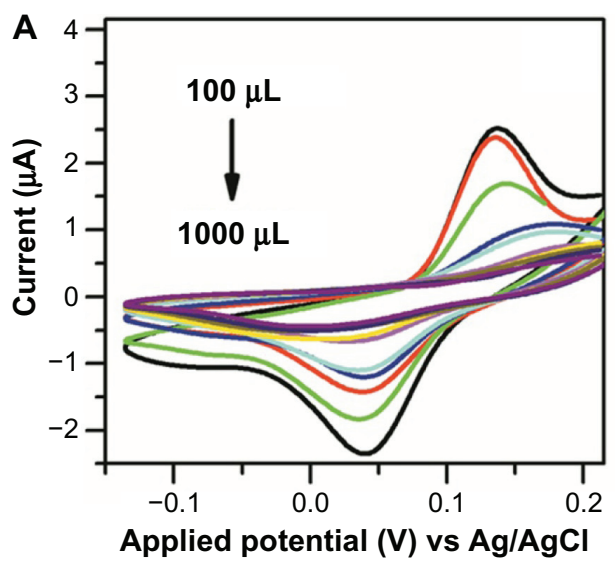

novel biosensor platform. Although the density of pure glucose in urine samples is usually detected using a differential amperometric method, in the current study we used the CV method to ensure the electrochemical response (oxidationreduction) of the Au-PCB-FGO-GOx electrode for feasibility in different physiological fluids.

Figure 5A shows the CV of Au-PCB-FGO-GOx versus different glucosuric sample volumes ranging from 100 to $1000 \mu \mathrm{L}$. A linear relationship was observed between the glucosuric sample and the peak current of the FGOmodified electrode. It is worth noting that, in contrast with commercial $\mathrm{D}(+)$ glucose in phosphate-buffered solution (Figure 4D), the peak current for Au-PCB-FGO-GOx versus that of the glucosuric sample decreased in a linear manner (see Figure 5B). Similarly, an earlier report based on glucose oxidase immobilized on hexamine ruthenium (III) chloride $\left[\mathrm{Ru}\left(\mathrm{NH}_{3}\right)_{6}\right] \mathrm{Cl}_{3}$ containing a nitrocellulose strip for urine glucose monitoring has also demonstrated a linear decrease in peak current with increasing glucose density. ${ }^{50}$ Possible reasons accounting for the decrease in peak redox current with a respective volumetric increase in urine glucose samples might include mixing of analyte urine glucose sample in bulk electrolyte phosphate-buffered solution and its dependent electrochemical reaction at the Au-PCB-FGOGOx electrode that influences the concentration variation in oxidation of produced $\mathrm{H}_{2} \mathrm{O}_{2}$ and reduction of $\mathrm{O}_{2}$. In addition, air saturation in phosphate-buffered solution and the existence of interferents in the urine samples may have decreased the peak electrocatalytic current for detection of $\mathrm{H}_{2} \mathrm{O}_{2}$ and reduction of dissolved $\mathrm{O}_{2}$.

In order to evaluate the electrochemical response of the Au-PCB-FGO-GOx electrode against blood glucose, serum

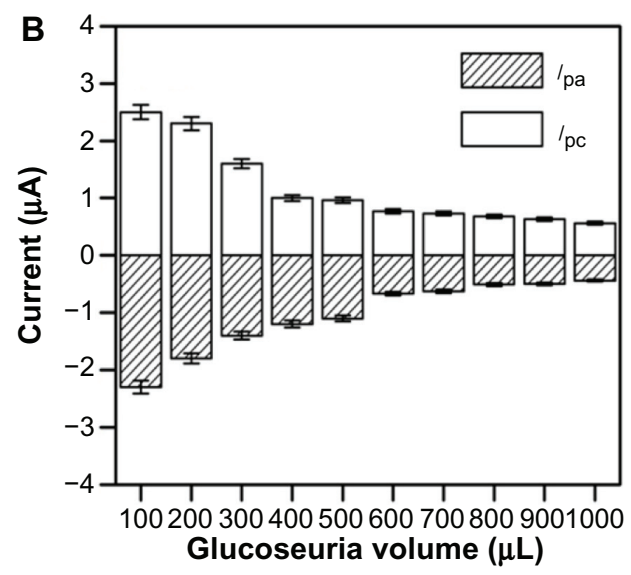

Figure 5 (A) CVs of Au-PCB-FGO-GOx against glucosuria sample (injected into supporting electrolyte) and (B) histogram of peak currents for Au-PCB-FGO/GOx electrode against glucosuria sample of different volumes from one diabetic patient, ensuring the linear relationship between the volume and peak current. Notes: Where error bars are not visible, they are smaller than the symbols. Scan rate: $50 \mathrm{mV} / \mathrm{s}$.

Abbreviations: CVs, cyclic voltammograms; Au-PCB-FGO-GOx, gold printed circuit board electrode modified with functionalized graphene oxide and glucose oxidase; $\mathrm{Ag} / \mathrm{AgCl}$, silver/silver chloride. 
samples from five diabetic patients with different glucose levels at 69, 144, 160, 183, and $256 \mathrm{mg} / \mathrm{dL}$ (measured using a commercial glucose sensor, Accu-Chek Performa, Roche, Basel, Switzerland) were tested. To mimic the analysis function of a commercial blood glucose sensor system, all serum glucose samples in the current study were modified separately on the surface of the Au-PCB-FGO-GOx electrode and measured for their electrochemical response. As can be seen from the CV measurement in Figure 6A, the lowest glucose concentration $(69 \mathrm{mg} / \mathrm{dL})$ among the five serum samples showed the lowest peak cathodic current at $0.83 \mu \mathrm{A}$, whereas the other glucose samples $(144,160,183$, and $256 \mathrm{mg} / \mathrm{dL})$ had higher peak cathodic currents $(1.11,2.28,2.61$ and $4.67 \mu \mathrm{A}$, respectively). This ensured a linear response against the serum glucose concentration and peak current obtained for the Au-PCB-FGO-GOx electrode. As discussed in the previous section, the peak redox current of Au-PCB-FGOGOx versus the serum glucose samples is observed to have a significant shift at different redox potentials (Figure 4D). However, the peak currents become relatively increased with increasing glucose concentration. These CVs (Figure 6A) are in relation to the commercial glucose CVs and are contrary to the urine samples. Although the same type of functional electrode comprised of Au-PCB-FGO-GOx has been utilized for the three different types of physiological glucose tested in this study, the individual response is distinctive. We suggest that the mechanism for the linear response of the peak current according to glucose concentration is highly dependent on the mode of electrochemical interaction, such as analyte molecules on the functional electrode or mixed into the electrolyte solution. The good concordance between detection at the commercial and clinical levels (glucosuria

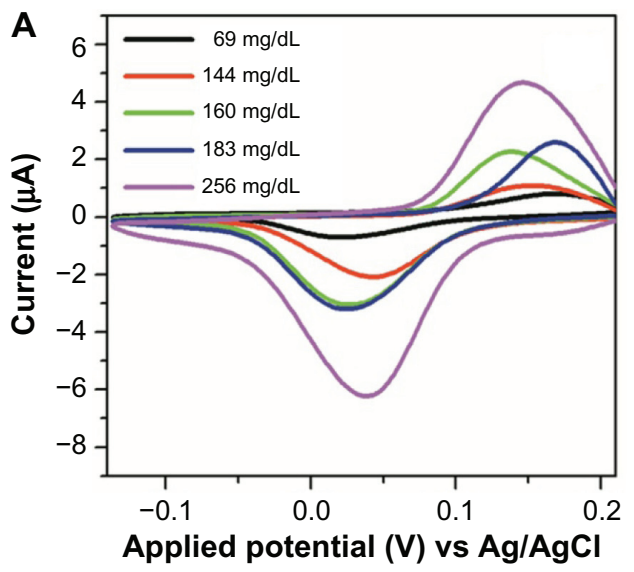

and serum glucose) confirms the reliability of our advanced hybrid material (FGO)-based electrochemical sensor. This method provides a novel sensitive platform for the development of an FGO-based electrochemical biosensor for clinical detection of glucose levels. Furthermore, the durability of our Au-PCB-FGO-GOx electrode for glucose detection in human serum was analyzed at the time of preparation (Figure S4A) and after 50 days of storage at $4^{\circ} \mathrm{C}$ (Figure S4B). As can be seen, the peak redox current intensity after the electrode had been incubated for 50 days is slightly decreased in comparison with that on the day of preparation; however, no fundamental change was observed in the position of the peak redox potential. This ensures the stability and binding efficiency of the active material modified on the electrode. As emphasized previously, the redox wave generation is attributed to the presence of MPHs on the surface of the graphene oxide. Unlike preparation of a conventional graphene oxide nanocomposite, ie, by physical adsorption, the present work involved a covalent reaction between silanized MPHs and carboxylic acid groups in graphene oxide, ${ }^{25}$ and the resulting chemical bonds enhanced the long-term binding affinity of MPHs at the graphene oxide-electrode interface. The high molecular weight PEG layer on the surface of the metalloid composite preserves the binding and bioactivity of glucose oxidase. ${ }^{37}$ Further, oxygen plasma pretreatment provides an excellent hydrophilic nature on the bare Au-PCB substrate which is also mediated by the strong affinity between the oxygenated graphene nanosheets and the Au-PCB substrate. Taken together, all these factors resulted in long-term stability of the glucose oxidase-modified Au-PCB-FGO electrode and provided better durability for studying the clinical glucose sample.

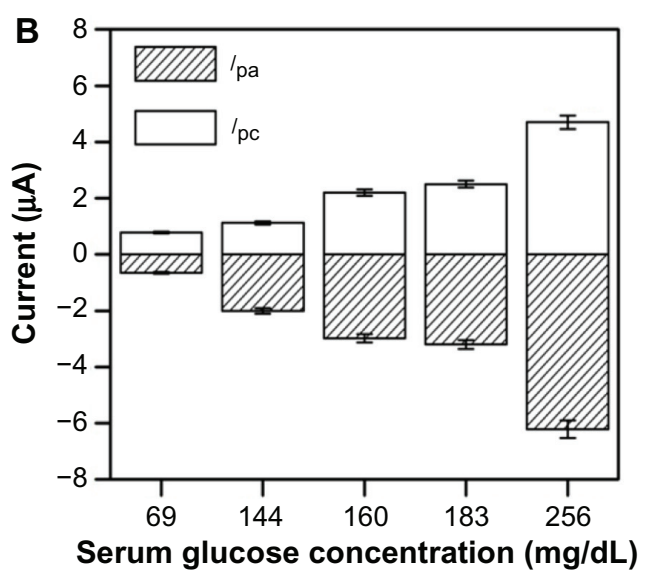

Figure 6 (A) CVs of Au-PCB-FGO-GOx against serum glucose samples (modified on electrode) from diabetic patients and (B) histogram of peak currents for Au-PCB-FGO/ GOx electrode against serum samples from low to high concentration from different diabetic patients.

Notes: Where error bars are not visible, they are smaller than the symbols. Scan rate: $50 \mathrm{mV} / \mathrm{s}$.

Abbreviations: CVs, cyclic voltammograms; Au-PCB-FGO-GOx, gold printed circuit board electrode modified with functionalized graphene oxide and glucose oxidase; $\mathrm{Ag} / \mathrm{AgCl}$, silver/silver chloride. 


\section{Conclusion}

In summary, an electrochemical biosensor based on $\mathrm{Ag} @$ $\mathrm{SiO}_{2}$-PEG (MPH) functionalized with graphene oxide was fabricated for glucose detection. Our electrochemical investigations demonstrate that functionalized graphene oxide provided a suitable electrocatalytic platform for glucose detection, which greatly facilitated electron exchange between the immobilized glucose oxidase enzyme and the electrode, and increased the detection ability for commercial and clinical glucose concentrations at multiple concentration ranges. Furthermore, properties such as inherent redox wave generation in phosphate-buffered solution and a hybrid nanoenvironment such as metal $(\mathrm{Ag})$, non-metal $\left(\mathrm{SiO}_{2}\right)$, and polymer (PEG) on the surface of novel graphene oxide make FGO a promising candidate for construction of other biosensor platforms.

\section{Acknowledgment}

This research was supported by grants from the Ministry of Knowledge and Economy (10039863) to KSYun and the Ministry of Knowledge and Economy (10032112) to M-HLee.

\section{Disclosure}

The authors report no conflicts of interest in this work.

\section{References}

1. Uenoyama H, Nankai S. In: Aizawa M, editor. Chemical Sensor Technology. Tokyo, Japan: Kodansha Ltd; 1994;5:177-185.

2. Cui G, Kim SJ, Choi S, et al. A disposable amperometric sensor screen printed on a nitrocellulose strip: a glucose biosensor employing lead oxide as an interference-removing agent. Anal Chem. 2000;72:1925-1929.

3. Miyashita M, Ito N, Ikeda S, et al. Development of urine glucose meter based on micro-planer amperometric biosensor and its clinical application for self-monitoring of urine glucose. Biosens Bioelectron. 2009;24: 1336-1340.

4. Nekrassova O, Lawrence NS, Compton RG. Analytical determination of homocysteine: a review. Talanta. 2003;60:1085-1095.

5. Nekrassova O, Lawrence NS, Compton RG. Selective electroanalytical assay for cysteine at a boron doped diamond electrode. Electroanalysis. 2004;16:1285-1291.

6. Sun TP, Shieh, HL, Ching CTS, et al. Carbon nanotube composites for glucose biosensor incorporated with reverse iontophoresis function for noninvasive glucose monitoring. Int J Nanomedicine. 2010;5: 343-349.

7. Schreier TM, Rach JJ, Howe GE. Efficacy of formalin, hydrogen peroxide, and sodium chloride on fungal-infected rainbow trout eggs. Aquaculture. 1996;140:323-331.

8. Ndamanisha JC, Bai J, Qi B, et al. Application of electrochemical properties of ordered mesoporous carbon to the determination of glutathione and cysteine. Anal Biochem. 2009;386:79-84.

9. Maleki N, Safavi A, Sedaghati F, et al. Efficient electrocatalysis of L-cysteine oxidation at carbon ionic liquid electrode. Anal Biochem. 2007;369:149-153.

10. Zhang D, Zhang K, Yao YL, et al. Multilayer assembly of Prussian blue nanoclusters and enzyme-immobilized poly(toluidine blue) films and its application in glucose biosensor construction. Langmuir. 2004;20: 7303-7307.
11. Veerapandian M, Subbiah R, Lim GS, et al. Copper-glucosamine microcubes: synthesis, characterization, and C-reactive protein detection. Langmuir. 2011;27:8934-8942.

12. Park HS, Park TJ, Huh YS, et al. Immobilization of genetically engineered fusion proteins on gold-decorated carbon nanotube hybrid films for the fabrication of biosensor platforms. J Colloid Interf Sci. 2010;350: 453-458.

13. Feng D, Wang F, Chen Z. Electrochemical glucose sensor based on one-step construction of gold nanoparticle-chitosan composite film. Sens Actuators B Chem. 2009;138:539-544.

14. Joung D, Chunder A, Zhai L, et al. Space charge limited conduction with exponential trap distribution in reduced graphene oxide sheets. Appl Phys Lett. 2010;97:093105.

15. Krishnamoorthy K, Mohan R, Kim SJ. Graphene oxide as a photocatalytic material. Appl Phys Lett. 2011;98:244101.

16. Krishnamoorthy K, Veerapandian M, Mohan R, et al. Investigation of Raman and photoluminescence studies of reduced graphene oxide sheets. Appl Phys A. 2012;106:501-506.

17. Pimenta MA, Dresselhaus G, Dresselhaus MS, et al. Studying disorder in graphite-based systems by Raman spectroscopy. Phys Chem Chem Phys. 2007;9:1276-1291.

18. Dreyer DR, Park SJ, Bielawski CW, et al. The chemistry of graphene oxide. Chem Soc Rev. 2010;39:228-240.

19. Shan CS, Yang HF, Han DX, et al. Graphene/AuNPs/chitosan nanocomposites film for glucose biosensing. Biosens Bioelectron. 2010;25:1070-1074.

20. Wu P, Shao Q, Hu Y, et al. Direct electrochemistry of glucose oxidase assembled on graphene and application to glucose detection. Electrochim Acta. 2010;55:8606-8614.

21. Zhang Y, Sun X, Zhu L, et al. Electrochemical sensing based on graphene oxide/Prussian blue hybrid film modified electrode. Electrochim Acta. 2011;56:1239-1245.

22. Ashok Kumar S, Cheng HW, Chen SM, et al. Preparation and characterization of copper nanoparticles/zinc oxide composite modified electrode and its application to glucose sensing. Mater Sci Eng CMater Biol Appl. 2010;30:86-91.

23. Veerapandian M, Yun KS. Ultrasonochemical-assisted fabrication and evaporation-induced self-assembly (EISA) of $\mathrm{POSS}_{-} \mathrm{SiO}_{2} @ \mathrm{Ag}$ core/ ABA triblock copolymer nanocomposite film. Polymer Composites. 2010;31:1620-1627.

24. Veerapandian M, Yun KS. Ultrasonochemically conjugated metalloid/triblock copolymer nanocomposite and subsequent thin solid laminate growth for surface and interface studies. Langmuir. 2010;26:14216-14222.

25. Veerapandian M, Lee MH, Krishnamoorthy K, et al. Synthesis, characterization and electrochemical properties of functionalized graphene oxide. Carbon. 2012;50:4228-4238.

26. Hirata M, Gotou T, Horiuchi S, et al. Thin-film particles of graphite oxide 1: high-yield synthesis and flexibility of the particles. Carbon. 2004;42:2929-2937.

27. Veerapandian M, Lim SK, Nam HM, et al. Glucosamine-functionalized silver glyconanoparticles: characterization and antibacterial activity. Anal Bioanal Chem. 2010;398:867-876.

28. Jiang $\mathrm{G}, \mathrm{Lin} Z$, Chen $\mathrm{C}$, et al. $\mathrm{TiO}_{2}$ nanoparticles assembled on graphene oxide nanosheets with high photocatalytic activity for removal of pollutants. Carbon. 2011;49:2693-2701.

29. Pittet P, Lu GN, Galvan JM, et al. PCB technology-based electrochemiluminescence microfluidic device for low-cost portable analytical systems. IEEE Sens J. 2008;8:565-571.

30. Ghindilis AL, Atanasov P, Wilkins E. Enzyme-catalyzed direct electron transfer: fundamentals and analytical applications. Electroanalysis. 1997;9:661-674

31. Shan CS, Yang HF, Song JF, et al. Direct electrochemistry of glucose oxidase and biosensing for glucose based on graphene. Anal Chem. 2009;81:2378-2382.

32. Shao Y, Wang J, Wu H, et al. Graphene based electrochemical sensors and biosensors: a review. Electroanalysis. 2010;22:1027-1036. 
33. Rao CN, Subrahmanyam KS, Ramakrishna Matte HS, et al. A study of the synthetic methods and properties of graphenes. Sci Technol Adv Mater. 2010;11:054502.

34. Wu W, Liu Z, Jauregui LA, et al. Wafer-scale synthesis of graphene by chemical vapor deposition and its application in hydrogen sensing. Sens Actuators B Chem. 2010;150:296-300.

35. Rao CN, Ramakrishna Matte HS, Subrahmanyam KS. Synthesis and selected properties of graphene and graphene mimics. Acc Chem Res. June 27, 2012. [Epub ahead of print.]

36. Lu R, Terrones M. Towards new graphene materials: doped graphene sheets and nanoribbons. Mat Lett. 2012;78:209-218.

37. Sung WJ, Bae YH. A glucose oxidase electrode based on polypyrrole with polyanion/PEG/enzyme conjugate dopant. Biosens Bioelectron. 2003;18:1231-1239.

38. Wang GF, Wang W, Wu JF, et al. Self assembly of a silver nanoparticles modified electrode and its electrocatalysis on neutral red. Microchim Acta. 2009;164:149-155.

39. Choi YJ, Luo T-JM. Electrochemical properties of silver nanoparticle doped aminosilica nanocomposite. Int J Electrochem. 2011;404937: $1-6$.

40. Guo L, Nie J, Du B, et al. Thermoresponsive polymer-stabilized silver nanoparticles. J Colloid Interface Sci. 2008;319:175-181.

41. Wang GF, Li MG, Gao YC, et al. Amperometric sensor used for determination of thiocyanate with a silver nanoparticles modified electrode. Sensors. 2004;4:147-155.

42. Chiu JY, Yu CM, Yen MJ, et al. Glucose sensing electrodes based on a poly(3,4-ethylenedioxythiophene)/Prussian blue bilayer and multiwalled carbon nanotubes. Biosens Bioelectron. 2009;24:2015-2020.
43. O’Halloran MP, Pravda M, Guilbault GG. Prussian blue bulk modified screen-printed electrodes for $\mathrm{H}_{2} \mathrm{O}_{2}$ detection and for biosensors. Talanta. 2001;55:605-611.

44. Wu S, Zhao H, Ju H, et al. Electrodeposition of silver-DNA hybrid nanoparticles for electrochemical sensing of hydrogen peroxide and glucose. Electrochem Commun. 2006;8:1197-1203.

45. Vijayalakshmi A, Karthikeyan R, Berchmans S. Nonenzymatic reduction of hydrogen peroxide produced during the bioelectrocatalysis of glucose oxidase on urchin-like nanofibrillar structures of cu on au substrates. J Phys Chem C Nanomater Interfaces. 2010;114:22159-22164.

46. Wang Z, Liu S, Wu P, et al. Detection of glucose based on direct electron transfer reaction of glucose oxidase immobilized on highly ordered polyaniline nanotubes. Anal Chem. 2009;81:1638-1645.

47. Xue MH, Xu Q, Zhou M, et al. In situ immobilization of glucose oxidase in chitosan-gold nanoparticle hybrid film on Prussian blue modified electrode for high-sensitivity glucose detection. Electrochem Commun. 2006;8:1468-1474.

48. Xian YZ, Hu Y, Liu F, et al. Template synthesis of highly ordered Prussian blue array and its application to the glucose biosensing. Biosens Bioelectron. 2007;22:2827-2833.

49. Niewoehner CB. Endocrine Pathophysiology, 2nd ed. Raleigh, NC: Hayes Barton Press; 2004.

50. Park HD, Lee KJ, Yoon HR, et al. Design of a portable urine glucose monitoring system for health care. Comput Biol Med. 2005;35: 275-286. 


\section{Supplementary materials}

Table SI Shows the patient details in terms of their age, gender and glucose concentration measured by commercial sensor AccuChek $^{\circledR}$ from Roche (Basel, Switzerland)

\begin{tabular}{llll}
\hline $\begin{array}{l}\text { Diabetic patient } \\
\text { serum sample no }\end{array}$ & $\begin{array}{l}\text { Patient } \\
\text { age }\end{array}$ & Gender & $\begin{array}{l}\text { Accu-Chek performa } \\
\text { (Roche) measured } \\
\text { glucose concentration }\end{array}$ \\
\hline $\mathrm{I}$ & 68 & $\mathrm{~F}$ & $69 \mathrm{mg} / \mathrm{dL}$ \\
2 & 49 & $\mathrm{~F}$ & $144 \mathrm{mg} / \mathrm{dL}$ \\
3 & 69 & $\mathrm{M}$ & $160 \mathrm{mg} / \mathrm{dL}$ \\
4 & 66 & $\mathrm{M}$ & $183 \mathrm{mg} / \mathrm{dL}$ \\
5 & 43 & $\mathrm{M}$ & $256 \mathrm{mg} / \mathrm{dL}$ \\
\hline
\end{tabular}

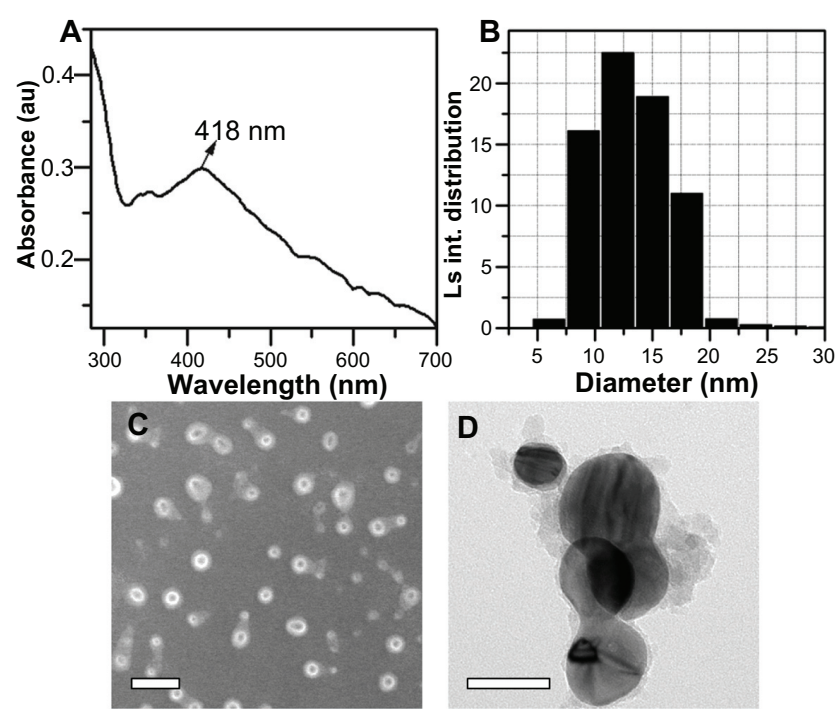

Figure SI (A) Ultraviolet-visible absorbance spectrum, and (B) particle size distribution histogram of metalloid polymer hybrid nanoparticles (Ag@SiO - -PEG) from electrophoretic light scattering measurements. (C) Field emission scanning electron microscopic (scale bar $30 \mathrm{~nm}$ ) and (D) high resolution transmission electron microscopic images (scale bar $10 \mathrm{~nm}$ ) of metalloid polymer hybrid nanoparticles. Abbreviations: $\mathrm{Ag@SiO}$-PEG, silver-silica coated with polyethylene glycol; LS int., light scattering intensity.
A

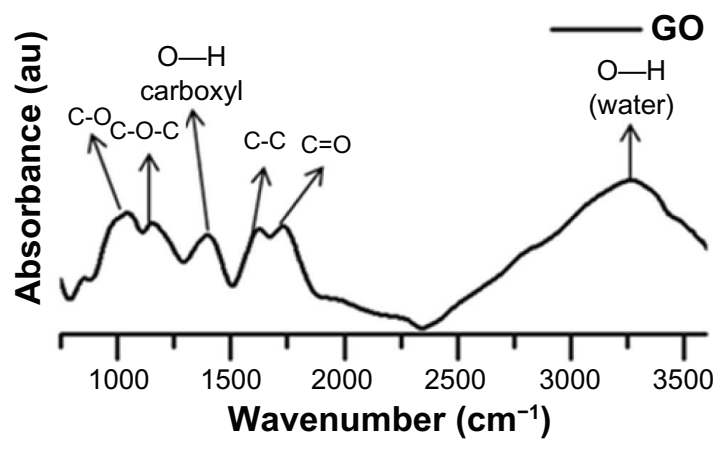

B

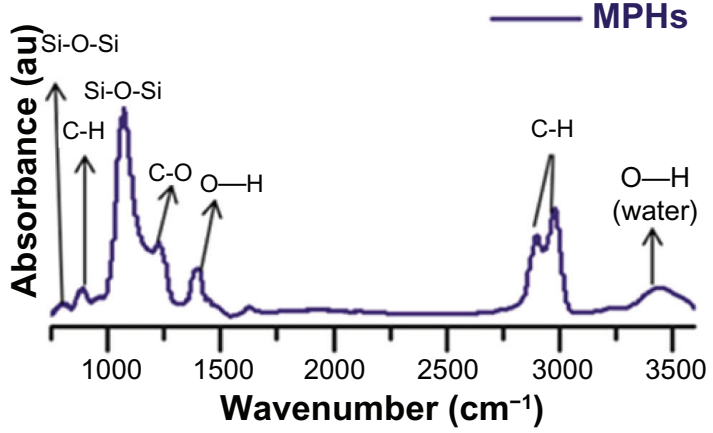

C

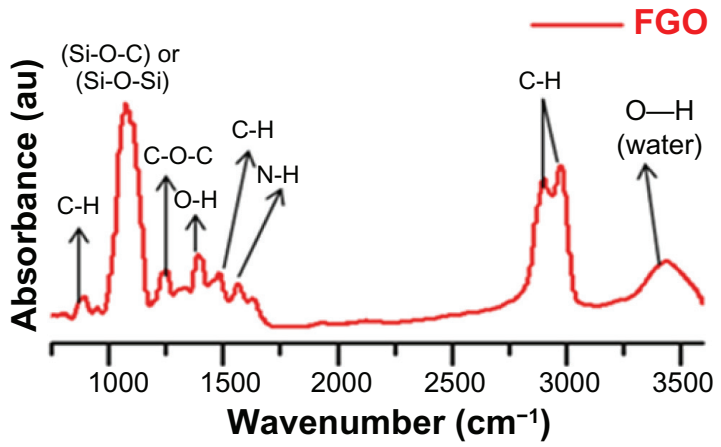

Figure S2 Fourier transform infrared absorbance spectra of (A) GO, (B) MPHs, and (C) FGO.

Abbreviations: GO, graphene oxide; MPHs, metalloid polymer hybrid nanoparticles; FGO, functionalized graphene oxide. 
A

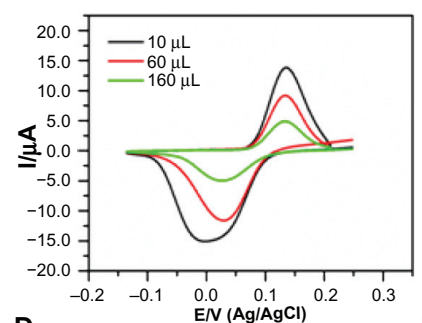

D

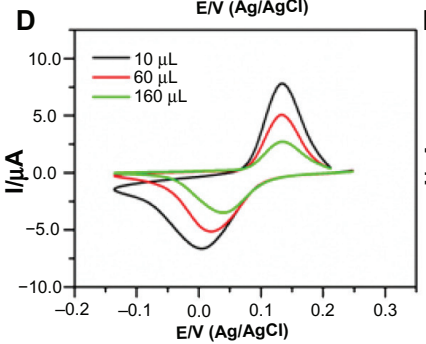

B

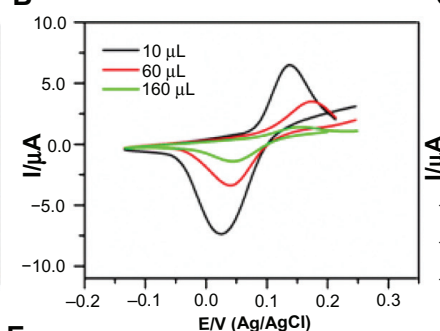

C
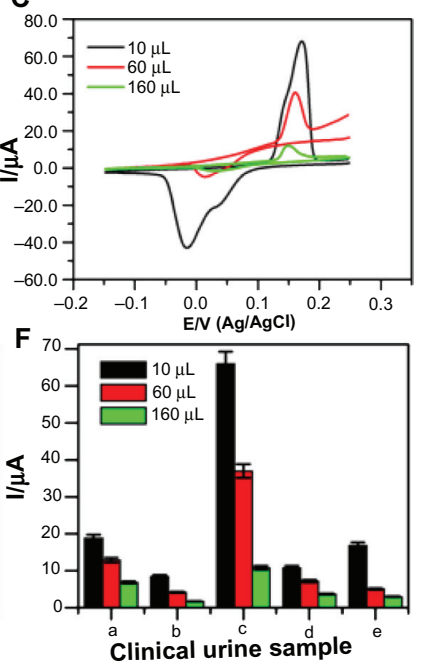

Figure S3 CVs of Au-PCB-FGO-GOx versus different clinical glucosuria samples (urine samples from five patients named A-E) at volumes of 10 , 60 , and I60 $\mu \mathrm{L}$ in phosphate-buffered solution. (F) Cathodic peak current histogram for five glucosuria samples.

Notes: This result shows the preliminary clinical diagnostics of urine samples from hyperglycemic patients. Scan rate: $50 \mathrm{mV} / \mathrm{s}$.

Abbreviations: CVs, cyclic voltammograms; Au-PCB-FGO-GOx, gold printed circuit board electrode modified with functionalized graphene oxide and glucose oxidase; $\mathrm{Ag} / \mathrm{AgCl}$, silver/silver chloride.
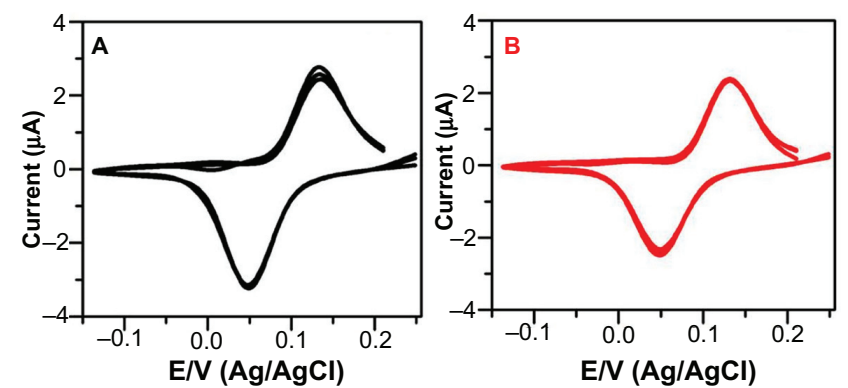

Figure S4 Multiple cycle CV segments of Au-PCB-FGO-GOx versus glucose serum sample (glucose concentration in serum $131 \mathrm{mg} / \mathrm{dL}$, measured by Accu-Chek ${ }^{\circledR}$ ) electrode measured on day 0 ( $\mathbf{A}$, black trace) and after 50 days (B, red trace) stored in refrigeration at $4^{\circ} \mathrm{C}$.

Note: Scan rate: $50 \mathrm{mV}$ per second.

Abbreviations: CV, cyclic voltammogram; Au-PCB-FGO-GOx, gold printed circuit board electrode modified with functionalized graphene oxide and glucose oxidase; $\mathrm{Ag} / \mathrm{AgCl}$, silver/silver chloride.

\section{Publish your work in this journal}

The International Journal of Nanomedicine is an international, peerreviewed journal focusing on the application of nanotechnology in diagnostics, therapeutics, and drug delivery systems throughout the biomedical field. This journal is indexed on PubMed Central, MedLine, CAS, SciSearch ${ }^{\circledR}$, Current Contents ${ }^{\circledR} /$ Clinical Medicine,
Journal Citation Reports/Science Edition, EMBase, Scopus and the Elsevier Bibliographic databases. The manuscript management system is completely online and includes a very quick and fair peer-review system, which is all easy to use. Visit http://www.dovepress.com/ testimonials.php to read real quotes from published authors. 